\title{
Design and Realization of Intelligent Website Traffic Monitoring System
}

\author{
Haiyun LIN ${ }^{1, a}$, Yujiao WANG ${ }^{1, b}$, Lianchang ZHANG ${ }^{1, c}$, Jianchun CAI ${ }^{1, d}$, \\ Xiaoyan WANG $^{1, e}$, Xiaoyuan Peng ${ }^{1, f}$ \\ ${ }^{1}$ Department of Physical Science and Technology, Kunming University \\ Yunnan, Kunming 650214, China \\ aemail: Ihy198@163.com , 'bCorresponding Author: e-mail:tjwyj817@163.com, \\ 'email: zhanglianchang123@163.com, 'email:584961280@163.com, \\ eemail: 1545593353@qq.com, 'email:pxy1028@126.com
}

Keywords: Intelligent website; traffic analysis; system structure; technical realization

\begin{abstract}
Through the analysis on the website traffic monitoring statistics technology, design and realize an intelligent website traffic monitoring system. The major modules have statistical generalization, latest visiting, source details, online statistics, page statistics, monthly statistics, second glance rate statistics, customer end statistics, calculator resetting, and exit management. This system reaches the pre-established requirements, which provides reference for the administrators to understand the website operation status promptly.
\end{abstract}

\section{Introduction}

With the development of the internet, the website not only pursues the website traffic, but also analyzes the website traffic statistics [1]. The website traffic analysis refers that, under the situation of obtaining the basic data of the website traffic, the website traffic includes what major statistical indexes. Carry out statistics and analysis on the related data, find out the rules of the user to visit the websites, combine these rules with the network marketing strategy, and then identity the possible problems in the current network marketing activities, and provide evidence for modifying or re-establishing the network marketing strategy [2]. The intelligent website traffic monitoring system is a set of statistical analysis software of the website traffic volume. Through arranging the installation and operation on the website, it is feasible to intuitively make statistics on the daily, monthly and annual traffic volume, IP visiting, and page view ranking, as well as which searcher it comes from. The result from the statistical analysis is intuitive and accurate, which enables the administrators to promptly understand the website operation status and visiting status.

\section{Technical Realization}

This software adopts B/S framework programming of ASP+ACCESS. The whole name is Active Server Pages, which is the development environment of WEB server terminal. It can generate and perform dynamic, interactive and high-performance WEB service application. ASP adopts VBscript (Java script) as its own developed language. ASP has strong compatibility, which is suitable for being applied in several browsers to view the pages. Besides, the language compatibility is high. ASP is compatible with all ActiveX Script language [3]. Except for combining with HTML, VBScript, Java Script, Active X server components, it can adopt "plug-in (component module)", and use the language provided by other manufacturers (Third Party). The privacy and security are so high. ASP can easily connect different databases through ODBC (Open Database Connectivity), such as Acess, Foxpro, dBase, Oracle, etc.

The database adopted for the software development is Microsoft Office Access, which is a database management system with the integration of the graphic user interface of the database engine and software development. The software developer and data architect can use Microsoft 
Access to develop application software. "Senior User" can use it to construct the software application. Like other office application, ACCESS supports Visual Basic macro-language. It is a programming language facing the objects. It can cite various objects, including DAO (data access objects), ActiveX data objects, and many other ActiveX modules. The visible object is to display the statement [4]. Their method and attribute refer that, under VBA programming environment, VBA code module can state and invoke Windows operation system function.

\section{Installation Deployment}

The software adopts B/S framework of ASP+ACCESS to make development. This system is deployed on Win2003 server, and it also needs to be allocated in IIS environment. Establish new stations on IIS, and allocate them. Firstly, it is necessary to fill in website to describe the website traffic statistics analysis system. IP address is IP and port number of the server. If the website domain name is applied, it is feasible to interpret DNS onto this IP address, and fill in DNS on the engine head of the website.

\section{System Structure}

The major function modules of the software are: statistical generalization, latest visiting, source details, online statistics, page statistics, monthly statistics, second glance rate statistics, customer end statistics, calculator resetting, and exit management.

The statistical generalization displays the whole statistical information of the website, including the statistical date, statistical days, number of online people, total traffic volume, daily traffic volume, monthly traffic volume, average daily access statistics, etc.

Display the latest access record, including the visitor's IP, used operation system, browser, access time, etc.

Specify the users' source details, and display whether the users enter from other websites or directly input the websites through the browser, as well as PV quantity, IP quantity, visited IP address, the last access time, etc.

Online statistics is to make statistics on the information of the online users, including the IP of the visitors, used operation system, browser, the starting time of the access, the last access time, skip resource, visited pages, etc.

The page statistics is to make statistics on the view amount of each page, proportion and other information.

The annual statistics is to make statistics on the users' access information monthly.

The second glance rate is to make statistics on the users' second glance status [5].

The customer end statistics is to make statistics on the customer end situation adopted by the visitors, including the browser, operation system, screen resolution ratio, etc.

The counter resetting is to reset the single item or all statistics. After the data is cleared up, it will not be recovered. Instead, it is necessary to work carefully, and the deletion can be performed through inputting the password of the administrators.

\section{Software code}

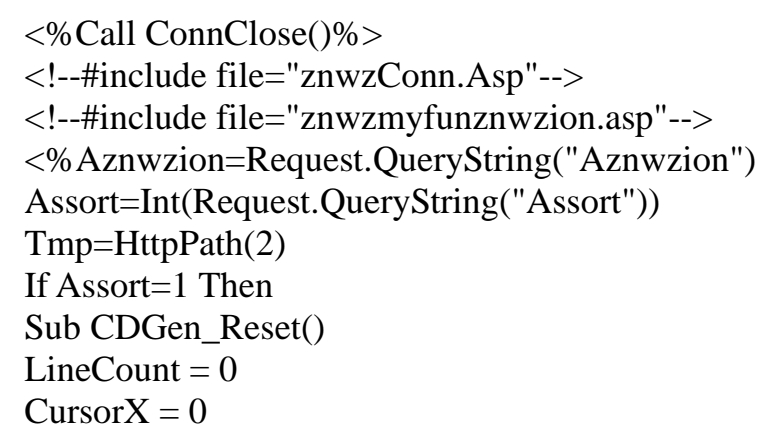




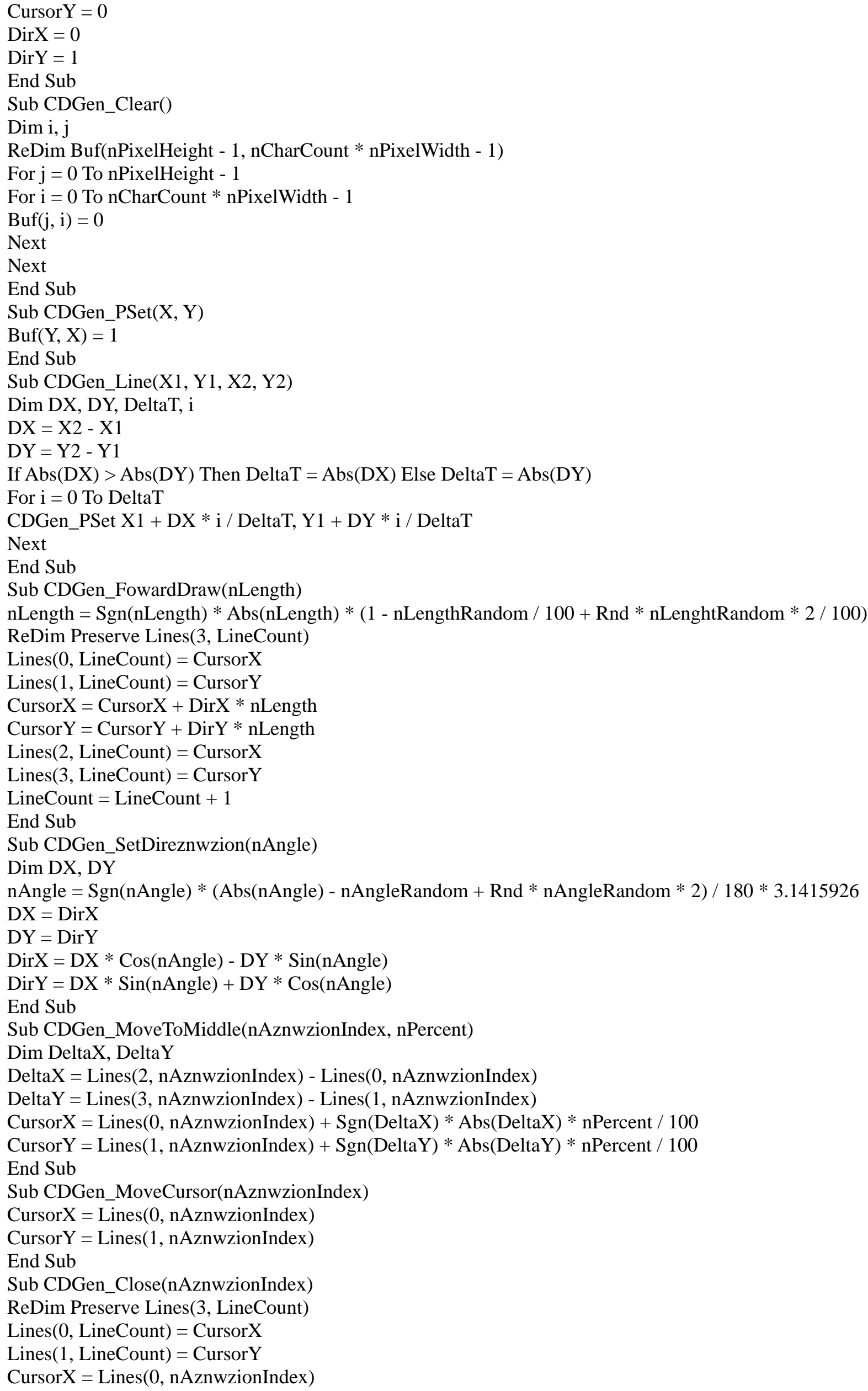




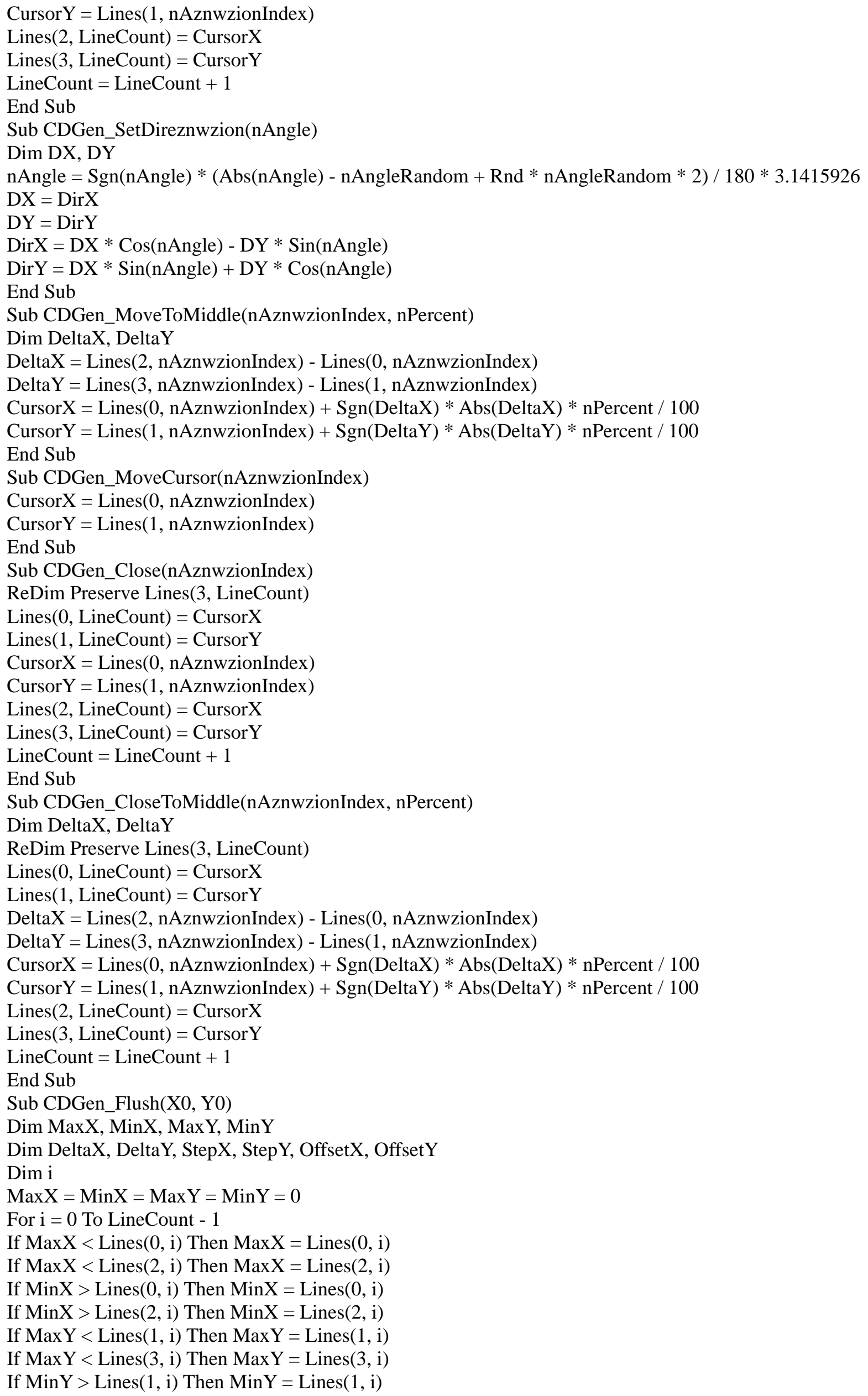




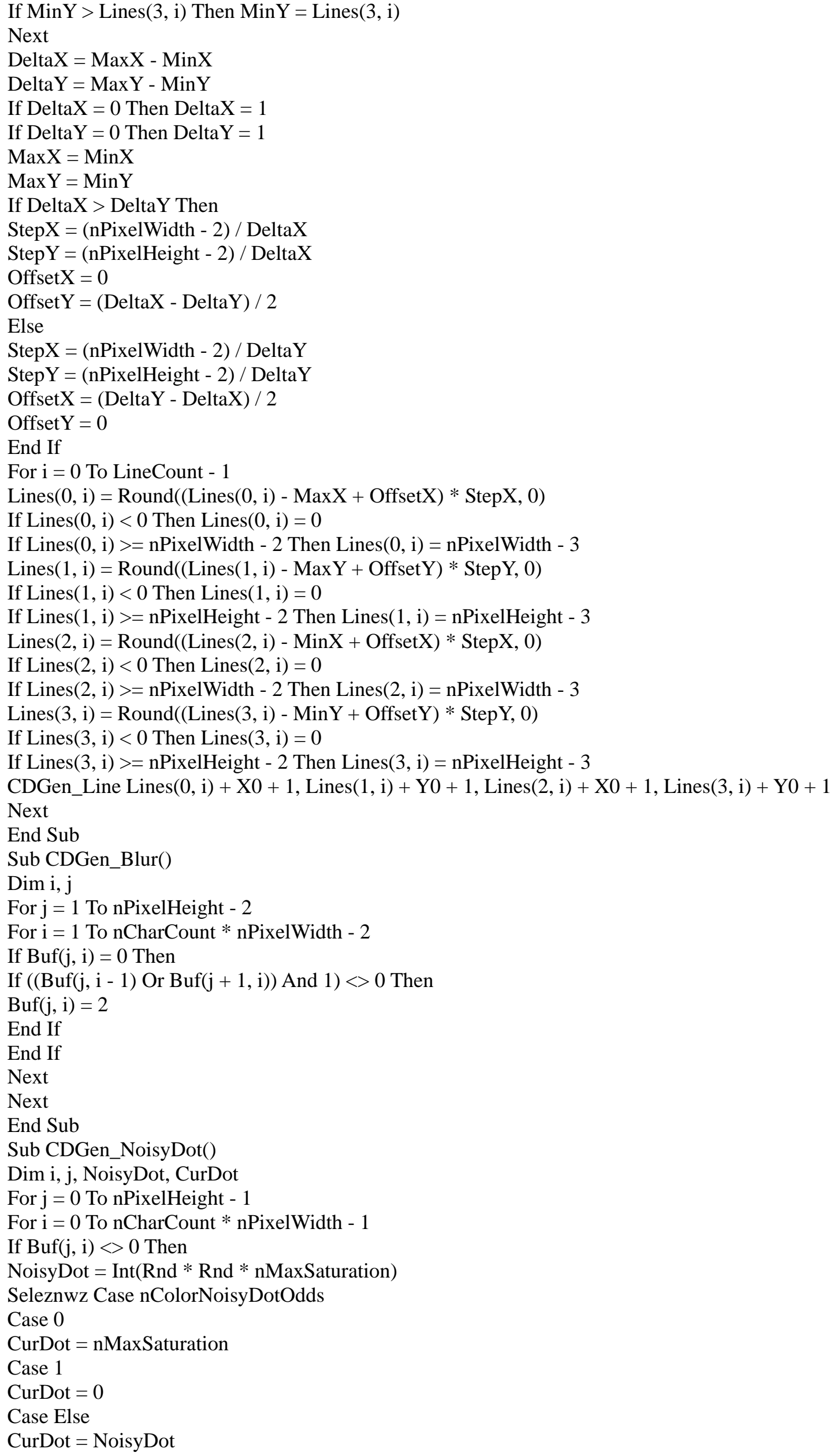




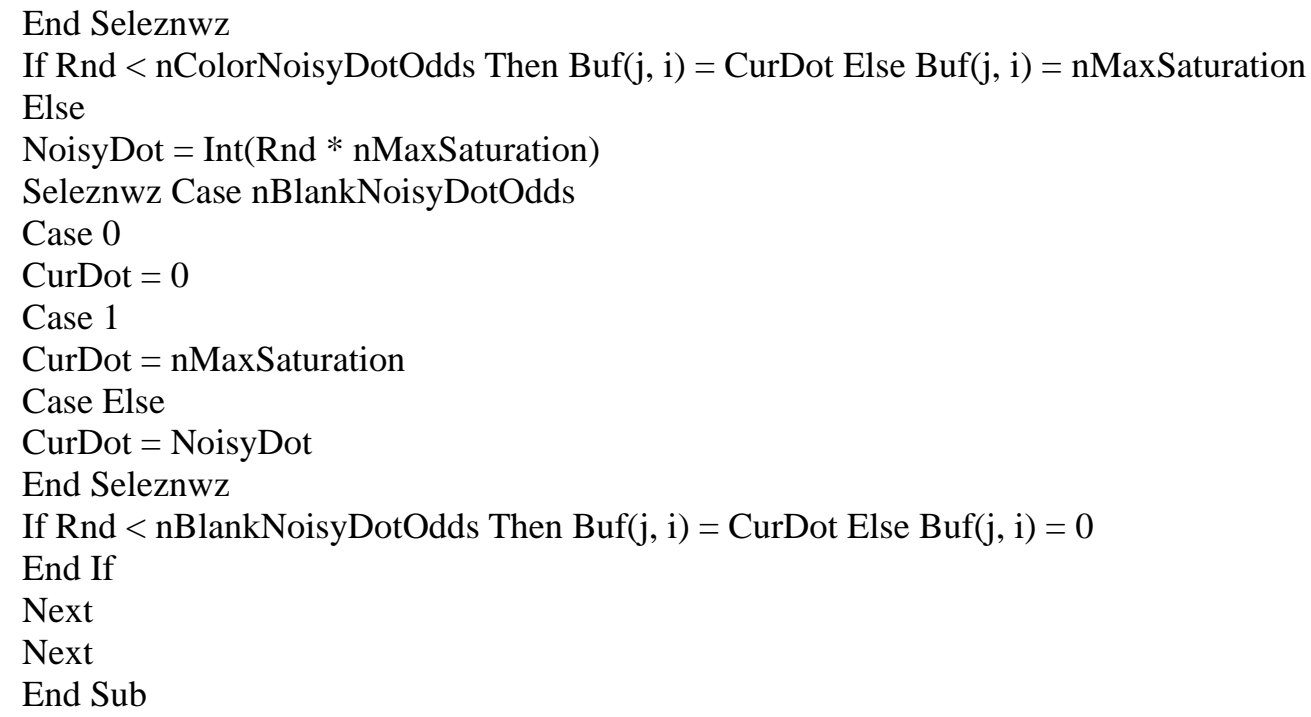

\section{Conclusion}

Through deploying installing and operating the intelligent website traffic monitoring system, it is feasible to intuitively make statistics on the daily, monthly and annual traffic volume, IP visiting, and page view ranking, as well as which searcher it comes from. The result from the statistical analysis is intuitive and accurate, which enables the administrators to promptly understand the website operation status and visiting status.

\section{References}

[1] Wang,X.,Abraham,A.,Smith,K.A.Intelligent Web Traffic Mining and Analysis. J. Network Comput. Appl . 2005, pp.231-234.

[2] Jonathan W Palmer.Web site usability, design, and performance metrics. Information Systems Research . 2002, pp.65-70

[3] Tarafdar M,Zhang J.Analyzing the Influence of Web Site Design Parameters on Web Site Usability. Information Resources Management Journal . 2005, pp.123-127.

[4] Charles Glommen,Blaise Barrelet.Internet website traffic flow analysis. UnitedStates Patent: US6393479B1 . 2002

[5] CAO Cai-feng, ZHANG Pei-ji, The Development and Realization of Website Visitor Volume Statistic Service System, Journal of Wuyi University, 2008, pp.64-68. 